\title{
Activité anti-hyperglycémique et antiradicalaire des extraits des fruits de Raphia gentiliana De Wild. (Arecaceae)
}

\author{
T.A. MASUNDA ${ }^{1}$, M.B. MBALA ${ }^{2}$, S.J. KAYEMBE ${ }^{2}$, B.F. LONGOMA ${ }^{1}$, \\ K.N. NGBOLUA ${ }^{2}$, D.S.T. TSHIBANGU ${ }^{2}$ et P.T. MPIANA ${ }^{2 *}$ \\ ${ }^{1}$ Institut Supérieur des Techniques Médicales de Kinshasa, RD Congo. \\ ${ }^{2}$ Faculté des Sciences, Université de Kinshasa, B.P. 190 Kinshasa, RD Congo. \\ *Auteur correspondant ; E-mail : ptmpiana@yahoo.fr; Tel : +243818116019
}

\section{RESUME}

Ce travail s'inscrit dans le cadre de la valorisation des extraits des tanins du fruit de Raphia gentiliana De wild, une plante médicinale utilisée comme hypoglycémiant par la population en République Démocratique Congo. L'étude est réalisée in vivo à partir d'un modèle animal constitué de 50 souris NMRI, chez qui on a provoqué une hyperglycémie temporaire par administration d'une solution de glucose (200 $\mathrm{mg} / \mathrm{ml}$ ). La réduction du pourcentage de glycémie des souris est mesurée deux heures après administration des extraits du fruit $(0,2 \mathrm{~g} / \mathrm{kg})$ de $R$. gentiliana (extraits de tanins et extraits totaux aqueux). Les valeurs de glycémie obtenues sont comparées avec un contrôle positif (glibenclamid). Les résultats obtenus montrent que l'extrait aqueux donne la valeur la plus élevée de réduction de la valeur de glycémie par rapport au contrôle négatif soit de 52,6\%. Une valeur comparable à celle du contrôle positif (52,5\%). Les extraits des tanins dans l'étherdiéthylique (EED), l'acétate d'éthyle (EAE) et le n-butanol/butanone (EBB) indiquent respectivement un pourcentage de réduction de glycémie de $51,1 \%$, 48,5\% et 7,5\%. La méthode de mesure de l'activité antiradicalaire est basée sur la détermination du pourcentage de réduction du radical DPPH en présence de l'extrait. Les propriétés antiradicalaires ont été mises en évidence par la mesure de la concentration efficace 50 $\left(\mathrm{CE}_{50}\right)$ et le calcul de l'indice de l'efficacité antiradicalaire (EAR). Les résultats obtenus donnent les valeurs de $\mathrm{CE}_{50}$ et EAR suivantes pour les différents extraits : Extrait aqueux (EAQ) : $72 \mathrm{mg}_{\text {extraits }} / \mathrm{mg}_{\mathrm{DPPH}}$ et $1,5010^{-3}$; EED : $65 \mathrm{mg}_{\text {extraits }} / \mathrm{mg}_{\mathrm{DPPH}}$ et $1,5310^{-3}$; EAE : $60 \mathrm{mg}_{\text {extraits }} / \mathrm{mg}_{\mathrm{DPPH}}$ et $1,6710^{-3} ; \mathrm{EBB}: 19 \mathrm{mg}_{\text {extraits }} / \mathrm{mg}_{\mathrm{DPPH}}$ et $12,0010^{-3}$. Il n'existe pas une corrélation directe entre l'activité antihyperglycémique et l'activité antiradicalaire.

() 2014 International Formulae Group. All rights reserved.

Mots clés : Raphia gentiliana, activité antioxydante, activité anti hyperglycémique, tanins.

\section{INTRODUCTION}

Le diabète insulino dépendant (type 1, diabète maigre ou diabète du jeune) aussi bien que non insulino dépendant (Type 2, diabète gras ou diabète de la personne âgée) est une maladie métabolique qui touche environ $2 \%$ de la population mondiale, soit environ 300 millions de personnes. Il est provoqué par un dérèglement du métabolisme glucidique qui est lié à une diminution du taux de l'insuline dans le sang (Ripsin et al., 2009).

Malgré d'importants progrès réalisés dans le traitement de cette maladie, des recherches sur les nouveaux médicaments 
contre les diabètes continuent du fait que de nombreux médicaments de synthèse ont montré leurs limites. Parmi les solutions préconisées, il y a la phytothérapie antidiabétique. Cette approche offre à ce jour, une alternative intéressante du fait de la découverte de plus en plus croissante d'extraits des plantes efficaces dans le traitement du diabète du type 2 (Zhang et al., 2000 ; Katemo et al., 2012).

Selon plusieurs études effectuées sur des modèles in vitro et in vivo, il a été constaté que les conditions d'hyperglycémies pouvaient provoquer l'installation d'une auto oxydation du glucose, d'une phosphorylation oxydative et d'une toxicité du glucose conduisant à la formation des radicaux libres impliquant un stress oxydatif (Riserus et al., 2009 ; Pitocco et al., 2014). A ce sujet, les polyphénols (tanins, anthocyanes, flavonoïdes, leucoanthocyanes) ont montré des résultats intéressants à la fois comme hypoglycémiants et antioxydants. Ces composés suscitent, en effet, de plus en plus de l'intérêt pour la prévention et le traitement de différentes maladies dont les cancers, les maladies inflammatoires, cardiovasculaires et neuro dégénératives (Lacopini et al., 2008).

Raphia gentiliana est une plante appartenant à la famille des Arecaceae. Ses fruits sont comestibles et sont réputés riches en lipides. Selon les enquêtes éthnobotaniques, une grande partie de la population de la République Démocratique du Congo (RDC) l'utilise comme aliment mais aussi comme plante médicinale pour ses vertus anti-diabétique et anti-inflammatoire (Lathan et Konda, 2006). Il est communément connu sous le nom de Makeke ou Nteke (Equateur), Masendé (Bas Congo), Balempâ ou Bakulu (Kinshasa et bandundu), Libondo (Nord Est et Sud Est de la RDC).

Le screening phytochimique du fruit de cette plante a révélé la présence des polyphénols, saponines, alcaloïdes et terpénoides. La détermination de l'indice et charge glycémique a montré des valeurs très faibles et son extrait aqueux a montré grâce à des études in vivo chez des souris, une activité antihyperglycémique intéressante (Mpiana et al., 2013).

Ce travail avait pour objectif l'étude de l'activité antiradicalaire et antihyperglycémique des différents extraits du fruit de Raphia gentiliana afin de voir s'il y a une corrélation entre ces deux activités.

\section{MATERIEL ET METHODES Matériel}

Le matériel végétal utilisé dans cette étude a été collecté à Bandundu (RDC) entre mars et juin 2013. L'indentification botanique a été faite par M Nlandu de l'institut national d'études et recherches agronomiques (INERA) de la faculté de sciences de l'université de Kinshasa. Le numéro voucher est Hombert 291.

\section{Préparation de l'extrait aqueux}

A $1 \mathrm{~g}$ de poudre de fruit de Raphia gentiliana a été ajouté $17 \mathrm{ml}$ d'eau distillée suivi de la macération pendant 24 heures dans un ballon rond à fond plat. La filtration a été faite à l'aide d'un papier filtre (Whatman ${ }^{\mathrm{TM}}$ 42) afin d'obtenir le macéré.

\section{Extraction de polyphénols}

L'extraction des polyphénols a été réalisée en utilisant un système de solvant Eau-Méthanol (30/70:V/V) à partir de la poudre délipidée en trois macérations de 50 gr/ $400 \mathrm{ml}$ du mélange eau-MeOH pendant 24 h. Le solvant est ensuite filtré avec du papier Wattman $\mathrm{N}^{\circ} 1$. La phase obtenue est évaporée à sec dans l'évaporateur rotatif.

\section{Extraction de tanins}

L'extraction de tanins a été réalisée par un mélange Eau-Acétone $(30 / 70: \mathrm{V} / \mathrm{V})$ en trois macérations sur la poudre dégraissée (50 gr/400 ml) pendant $24 \mathrm{~h}$. Avant chaque macération, on ajoute $4 \mathrm{ml}$ d'une solution de métabisulfite de sodium $(0,2 \%)$ pour éviter l'oxydation des tanins. Le solvant est ensuite filtré avec du papier Wattman et l'acétone est éliminée par distillation. La solution aqueuse obtenue est évaporée à sec sous agitation et chauffage modéré à $50{ }^{\circ} \mathrm{C}$ à l'aide d'un 
évaporateur rotatif. Le résidu sec est repris avec $200 \mathrm{ml}$ d'eau distillée. Une décantation de $24 \mathrm{~h}$ est nécessaire pour éliminer des éventuels dépôts. La solution aqueuse est alors soumise à différents affrontements (partition entre solvant); cette étape permet de séparer les tanins selon leur structure et leur degré de polymérisation. L'extraction avec l'éther diéthylique (EED) donne la fraction des tanins monomériques, celle avec l'acétate d'éthyle (EAE) donne la fraction des tanins dimériques et celle avec le butanol/butanone (EBB) donne la fraction des tanins tri et polymériques (Macheix 2005; Bruneton, 2009).

\section{Activité antiradicalaire}

L'activité antiradicalaire a été réalisée en utilisant le test au 2,2-diphényl-1picrylhydrazyle (DPPH). Les solutions méthanoliques de DPPH à différentes concentrations ont été obtenues à partir d'une solution de concentration $0,083 \mathrm{mg} / \mathrm{ml}$ dont on prélève des volumes successifs variant entre 3,975-3,850 $\mathrm{ml}$ et qu'on mélange avec différents volumes des solutions d'extraits à tester de concentration $1,33 \mathrm{mg} / \mathrm{ml}(0,025$ $0,150 \mathrm{ml}$ ) de façon à obtenir chaque fois $4 \mathrm{ml}$ de volume final.

Le mélange réactionnel est agité vigoureusement pendant 10 seconds. Le contenu est ensuite transféré dans la cuvette $\mathrm{du}$ spectrophotomètre pendant le temps nécessaire pour atteindre le plateau. A des intervalles de temps réguliers, les absorbances à $517 \mathrm{~nm}$ ont été lues et enregistrées contre le blanc à l'aide d'un spectrophotomètre UVVIS SAFAS Monaco 320.

Chaque test est répété trois fois en vue de déterminer les moyennes et les écarts types.

Le pourcentage de réduction des radicaux libre a été évalué par le rapport relatif de la concentration résiduelle $\mathrm{DPPH}_{\mathrm{t}} \mathrm{t}=$ Téq) restant en fin de cinétique par rapport à sa concentration initiale selon :

$\%[D P P H]=\frac{[D P P H]_{t} x 100}{[D P P H]_{0}}$
Le temps d'équilibre $\mathrm{T}_{\text {éq }}$ est déterminé à partir des courbes cinétiques. Il correspond au moment où le pourcentage de DPPH atteint le palier. La concentration efficace $\mathrm{CE}_{50}$, c'est-à-dire la concentration de l'extrait qui réduit $50 \%$ de $\mathrm{DPPH}$ et du temps $\mathrm{TCE}_{50}$ correspondant à cette concentration sont déductible par interpolation graphique à partir de la courbe du pourcentage de réduction de $\mathrm{DPPH}$ en fonction du rapport massique $\mathrm{Rm}$ (exprimée en mg d'antioxydant / g de DPPH). L'indice de l'efficacité antiradicalaire EAR a été calculé selon la relation :

$$
E A R=\frac{1}{C E_{50}\left(\frac{m g}{g D P P H}\right) \cdot T C E_{50}}
$$

\section{Evaluation de l'activité antihyperglycémique}

L'étude a été réalisée sur un model animal constitué de 50 souris NMRI (25 males et 25 femelles) soumis a une hyperglycémie temporaire par gavage d'une solution de glucose $(200 \mathrm{mg} / \mathrm{ml})$. Ces 50 souris ont été reparties en six groupes d'études ci-après : un $1^{\mathrm{er}}$ groupe de 5 souris pour le contrôle négatif (eau physiologique) un $2^{\text {ème }}$ groupe de 9 souris pour le contrôle positif avec glibenclamide $(10 \mathrm{mg} / \mathrm{kg})$ (Rani et al., 2014) et 4 groupes de 9 souris chacun pour le test avec les extraits aqueux et des tanins de $R$. gentiliana.

Le dosage de la glycémie a été effectué à l'aide d'un glucomètre SD check sur sang total prélevé au niveau de la queue.

\section{RESULTATS}

\section{Extraction des polyphénols et tanins de $\boldsymbol{R}$. gentiliana}

Le Tableau 1 donne la teneur en polyphénols et tanins totaux des fruits de $R$. gentiliana en général et celles des tanins extraits par différents solvants.

Il ressort de ce tableau que le fruit de $R$. gentiliana contient près de $19 \%$ de polyphénols et $13 \%$ des tanins totaux. Les tanins extraits par le système butanol/butanone permet d'atteindre une teneur de $6,3 \%$. 


\section{Activité antiradicalaire}

Les Figures 1 et 2 montrent les profils de la cinétique de réduction du DPPH en présence respectivement de l'extrait aqueux et de l'extrait éthéré des tanins des fruits de $R$. gentiliana.

Ces courbes montrent que la concentration en DPPH diminue avec le temps. Cette diminution est rapide au début de la réaction. La chute de la concentration en DPPH est plus prononcée pour des concentrations élevées en extraits de fruits indiquant une transformation massive des radicaux DPPH en molécules stables. Ces courbes atteignent un plateau où la concentration en DPPH ne varie plus avec le temps. Ce plateau est de plus en plus bas quand la concentration augmente.

Les Figures 3 et 4 donnent les courbes types de l'évolution de la concentration résiduelle de DPPH en fonction de la concentration en extrait de fruit de $R$. gentiliana. Ces courbes montrent que la concentration du radical DPPH baisse avec l'augmentation de la concentration en extraits de fruit de $R$. gentiliana.

Pour quantifier l'activité antioxydante on tire de ces courbes les valeurs des concentrations nécessaires pour réduire la concentration en DPPH à 50\%, le $\mathrm{CE}_{50}$.

Les paramètres caractéristiques de la cinétique de réduction de DPPH par les extraits des fruits de $R$. gentiliana sont présentés dans le Tableau 2.

\section{Activité antihyperglycémique des extraits des fruits de $R$. gentiliana sur les souris}

L'extrait aqueux des fruits de Raphia gentiliana ainsi que les différents extraits des tanins obtenus à partir de ces fruits ont été administrés aux souris NMRI pour voir leur effet antihyperglycémique. Les valeurs moyennes de glycémie des souris NMRI obtenues sont reprises dans le Tableau 3.

Pour mieux évaluer l'activité antihyperglycémique des extraits des fruits de Raphia gentiliana, il est nécessaire de mettre dans un même graphique, l'effet de l'extrait à côté de ceux des contrôles positif et négatifs. Les Figures 5 et 6 donnent à titre d'exemple les variations de la glycémie de souris soumis aux extraits aqueux et éther diéthylique par rapport à ceux soumis aux contrôles positif et négatif.

Tableau 1 : Teneur en polyphénols et tanins des fruits de $R$. gentiliana.

\begin{tabular}{lcc}
\hline Métabolite secondaire & Type d'extrait & Teneur (\%) \\
\hline Polyphénol & Eau/Méthanol & 18,9 \\
\hline Tanins selon leurs structures & Ether diéthylique & 3,2 \\
& Acétate d'éthyle & 3,6 \\
& butanol /butanone & 6,3 \\
\hline Tanins totaux & Eau/Acétone & 12,9 \\
\hline
\end{tabular}

Tableau 2 : Paramètres caractéristiques de la cinétique de réduction du DPPH.

\begin{tabular}{lcccc}
\hline Extrait & $\mathbf{C E}_{\mathbf{5 0} \text { (mgantiox/mgDPPH) }}$ & $\mathbf{T}_{\text {éq }}$ & $\mathbf{E A R}\left(\mathbf{x 1 0} \mathbf{0}^{-3}\right)$ & Classification \\
\hline EAQ & $72,0 \pm 1,2$ & $10,0 \pm 1,2$ & $1,50 \pm 0,02$ & Intermédiaire \\
EED & $65,4 \pm 1,0$ & $10,0 \pm 1,3$ & $1,53 \pm 0,03$ & Intermédiaire \\
EAE & $60,0 \pm 0,8$ & $10,0 \pm 1,1$ & $1,67 \pm 0,03$ & Intermédiaire \\
EBB & $19,4 \pm 0,3$ & $4,0 \pm 0,4$ & $12,00 \pm 2,01$ & Très élevé \\
Contrôle positif & $73,2 \pm 1,7$ & $10,0 \pm 0,9$ & $1,40 \pm 0,02$ & Intermédiaire \\
\hline
\end{tabular}


Tableau 3 : Valeurs moyennes de glycémies chez les souris soumises à l'étude.

\begin{tabular}{lllll}
\hline \multirow{2}{*}{ Groupe } & \multicolumn{4}{c}{ Concentration $(\mathbf{m g} / \mathbf{d l})$} \\
\cline { 2 - 5 } & GTO & GT6O & GT90 & GT120 \\
\hline Lot contr négatif & $133,4 \pm 0,1$ & $214,4 \pm 0,3$ & $201,5 \pm 0,2$ & $201,1 \pm 0,3$ \\
Lot contr positif & $133,4 \pm 0,1$ & $155,8 \pm 0,1$ & $117,4 \pm 0,2$ & $95,6 \pm 0,2$ \\
Lot EAQ & $133,4 \pm 0,1$ & $156,6 \pm 0,2$ & $119,2 \pm 0,1$ & $95,3 \pm 0,1$ \\
Lot EED & $133,4 \pm 0,1$ & $149,6 \pm 0,2$ & $121,2 \pm 0,1$ & $98,4 \pm 0,1$ \\
Lot EAE & $133,4 \pm 0,1$ & $151,4 \pm 0,1$ & $131,6 \pm 0,2$ & $103,5 \pm 0,1$ \\
Lot EBB & $133,4 \pm 0,1$ & $210,5 \pm 0,2$ & $200,6 \pm 0,1$ & $198,1 \pm 0,2$ \\
\hline \multicolumn{4}{l}{ Glycémie moyenne de souris à jeun : 133 mg/dl ; Dose essais $0,2 \mathrm{~g} / \mathrm{kg} ;$ Poids moyen $22 \mathrm{~g} ;$} \\
\multicolumn{4}{l}{ Dose contrôle positive (10mg / $\mathrm{kg})$. Contr : contrôle. }
\end{tabular}

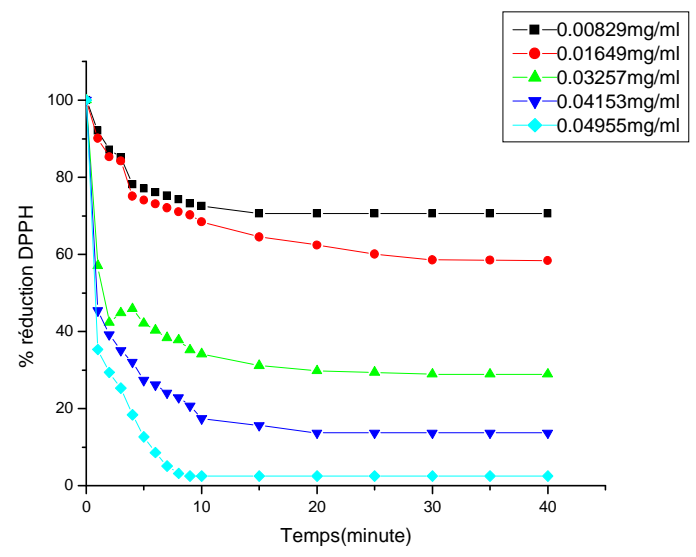

Figure 1: Variation du pourcentage de réduction du DPPH en fonction du temps en présence de l'extrait aqueux des fruits de $R$. gentiliana.

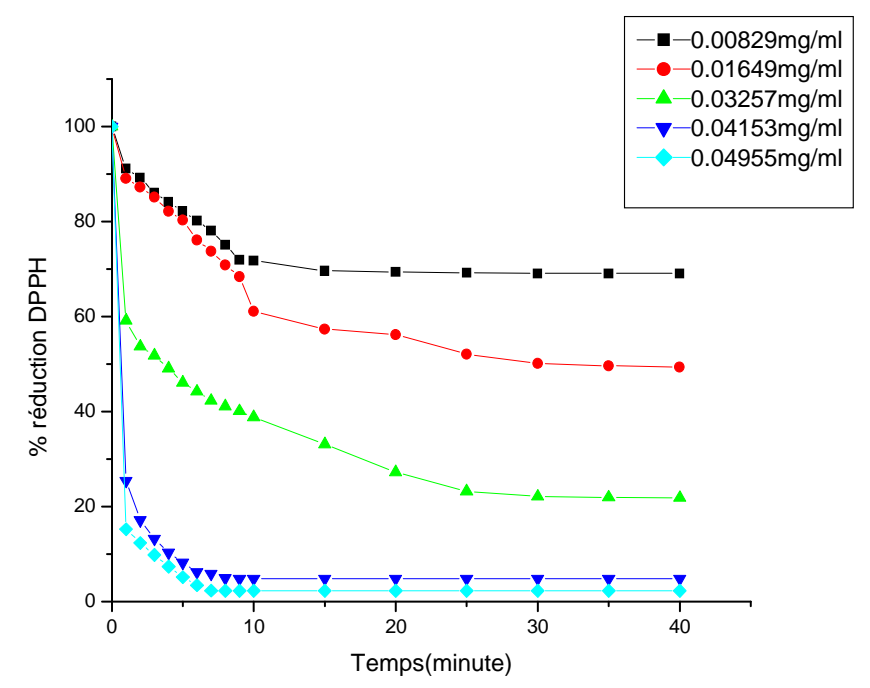

Figure 2: Variation du pourcentage de réduction du DPPH en fonction du temps en présence de l'extrait dans éther diéthylique des tanins de $R$. gentiliana. 


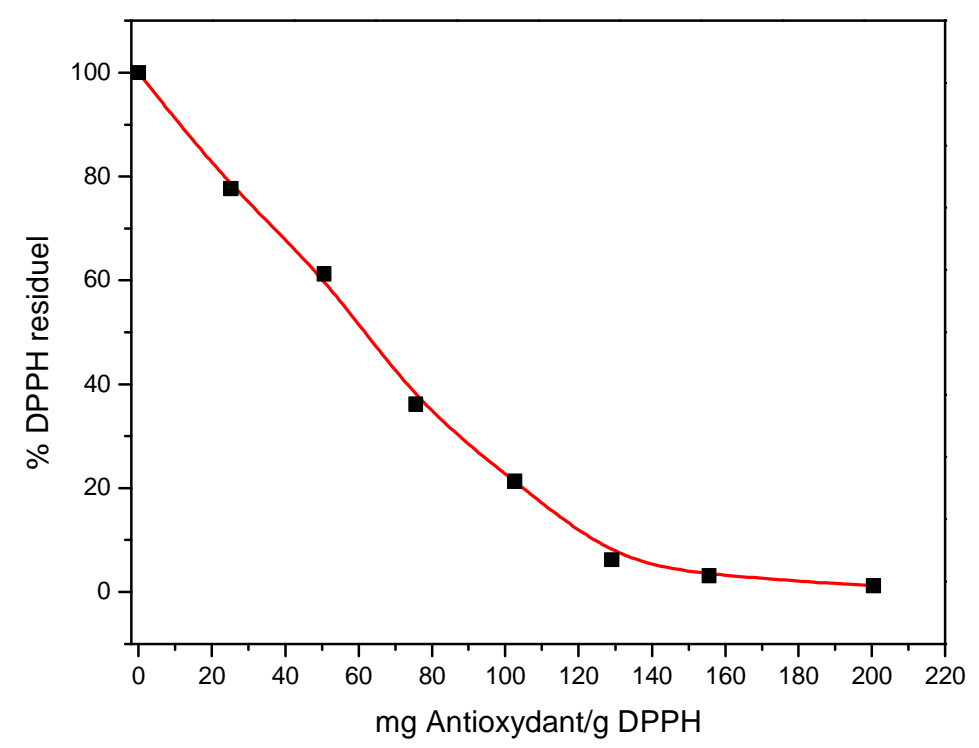

Figure 3: Variation du pourcentage de réduction du DPPH de EED en fonction du rapport massique.

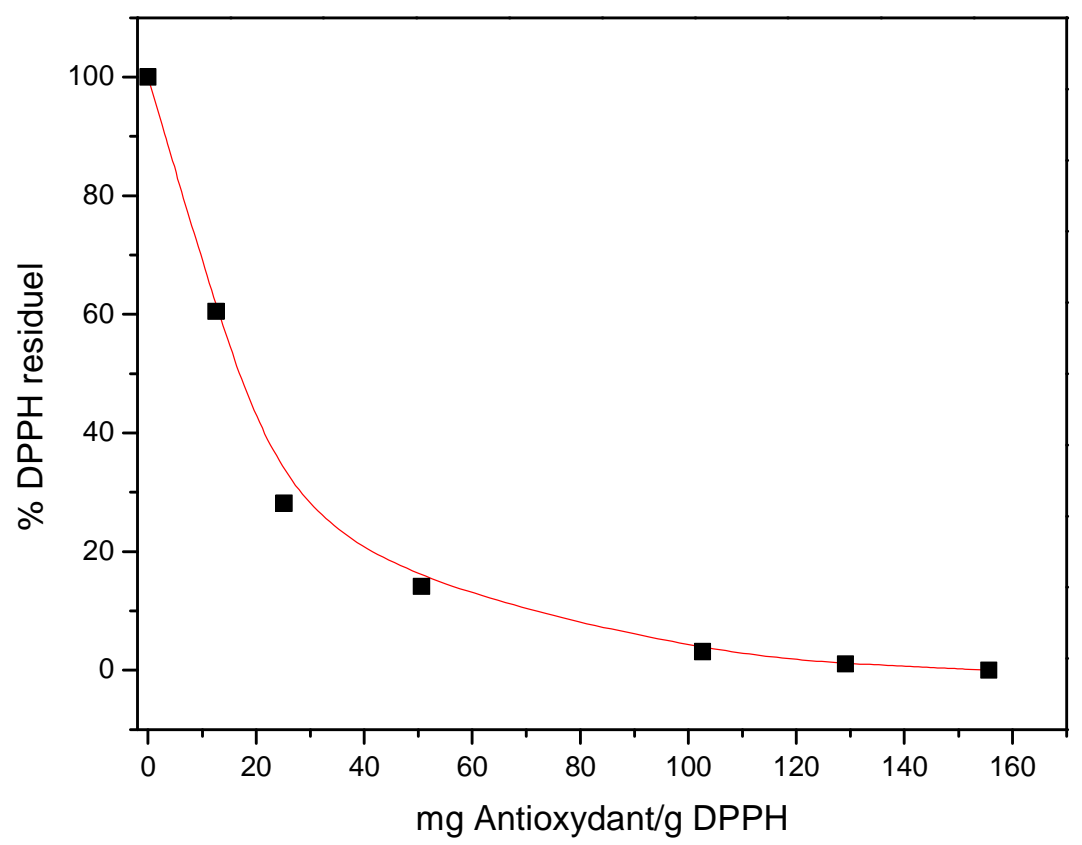

Figure 4: Variation du pourcentage de réduction du DPPH par le EBB en fonction du rapport massique. 


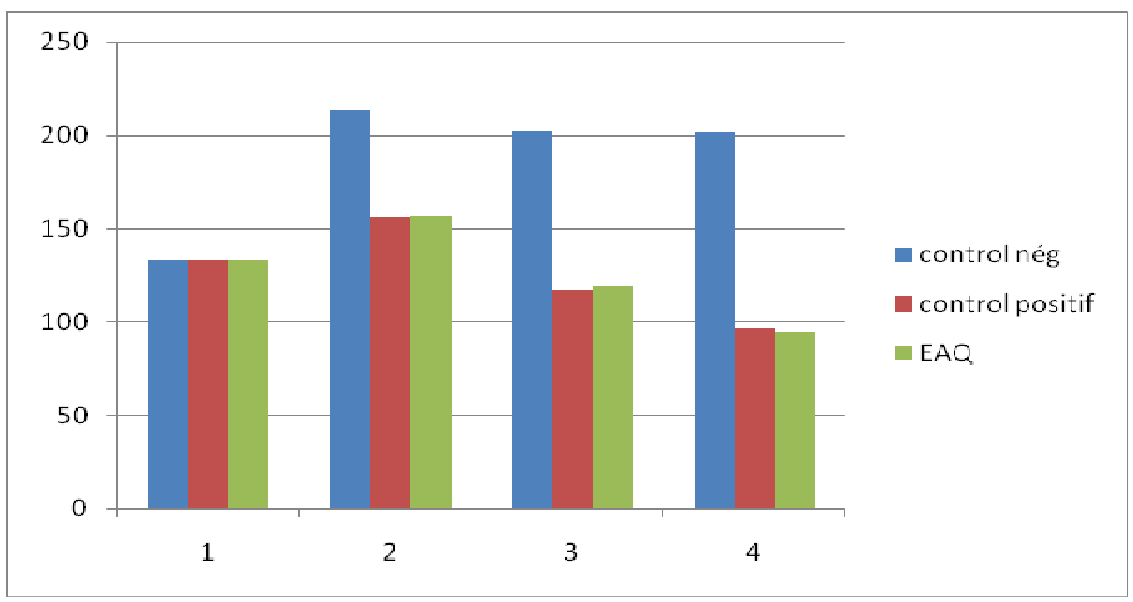

Figure 5 : Variation de la glycémie de souris soumis à l'extrait aqueux par rapport à ceux soumis aux contrôles positif et négatif.

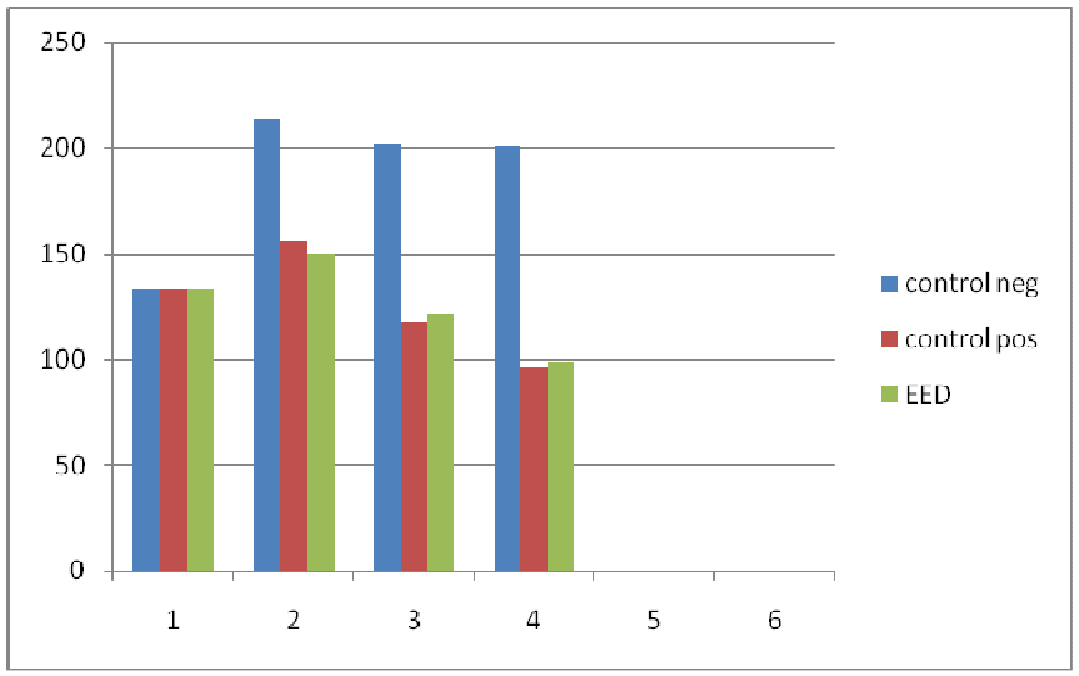

Figure 6 : Variation de la glycémie de souris soumis à l'extrait éther diétylique par rapport à ceux soumis aux contrôles positif et négatif.

\section{DISCUSSION}

\section{Activité antiradicalaire}

Le profil cinétique de la réduction du radical DPPH en présence des extraits de fruit de $R$. gentiliana montre que plus la concentration de l'antioxydant augmente, plus la réduction de pourcentage de DPPH est importante (Figures 1 et 2 ). Ce qui indique que cette activité antiradicalaire est dépendante du rapport massique $\mathrm{Rm}$ (mg d'antioxydant/g DPPH) (Muanda, 2010 ; Ngbolua et al., 2014 ).

La baisse de la concentration du radical DPPH indique que ce dernier est transformé en un autre produit par l'extrait de fruits traduisant ainsi l'activité antiradicalaire des extraits. En effet, il est connu que dans le cas des composés phénoliques, le mécanisme principal de réduction de la concentration du radical DPPH est le piégeage des radicaux libres par le transfert de l'atome $\mathrm{H}$ sur le 
DPPH qui se transforme en une molécule stable, le DPPHH (Prakash, 2007 ; Sharma, 2009).

Les valeurs de $\mathrm{CE}_{50}$ tirées des courbes présentant le pourcentage de réduction du radical DPPH en fonction du rapport massique (Figures 3 et 4) indiquent que l'extrait EBB a la valeur de concentration nécessaire pour réduire la concentration en DPPH à 50\% la plus basse soit $19,4 \mathrm{mg}$ d'extrait/mg DPPH. Ce comportement est confirmé par les valeurs de temps d'équilibre et d'indice de l'efficacité antiradicalaire (Tableau 2). Les valeurs d'indice EAR obtenues montrent l'ordre décroissant de l'activité antioxydante suivant : EBB> EAE> EED>EAQ >gliben clamid. L'extrait EBB a une activité antiradicalaire très élevée alors que les autres extraits y compris le témoin positif ont une activité intermédiaire. En effet, selon la classification proposée par Sanchez-Moreno et al. (1998), un composé a une activité antiradicalaire faible pour des valeurs de $\operatorname{EAR}<10^{-3}$, intermédiaire pour les valeurs de EAR comprises entre $10^{-3}-510^{-3}$, élevée pour les valeurs entre $5.10^{-3}-10^{-2}$; et très élevées pour EAR $>10^{-2}$.

L'étude réalisée par Sanchez-Moreno et son équipe sur des composés moléculaires a montré que l'acide gallique avait un EAR = 2,62 $10^{-3}$ (intermédiaire), l'acide tannique 0,57 $10^{-3}$ (faible) et l'acide ascorbique (Très élevé) avec EAR $=11,4410^{-3}$ (Sanchez Moreno et al., 1998). L'extrait EBB de fruit de $R$. gentiliana contenant les tanins polymériques a donc une activité antiradicalaire de même ordre que celle de l'acide ascorbique soit 12 $10^{-3}$.

En effet, les études sur la relation entre la structure chimique des composés phénoliques et leur capacité à piéger les radicaux libres ont montrés que l'activité antiradicalaire est dépendante du nombre; de la position et de la nature du substituant sur les cycles B et $\mathrm{C}$ (groupement hydroxylé; métaxylé; glycosylé) et du degré de polymérisation de ces composés. Cette activité croîtrait avec le degré de polymérisation pour les tanins (Scherer et Godoy, 2009).

\section{Activité antihyperglycémique}

A ce jour, des nombreuses plantes hypoglycémiantes ont été recensées. Les résultats sur des modèles animaux in vitro et in vivo ont montré que les extraits de plante (aqueux, polyphénoliques, alcaloïdiques et à saponines) pouvaient agir par divers mécanismes pour abaisser la glycémie. L'hyperglycémie pouvant être induit soit temporairement à partir d'une solution de glucose soit définitivement par contact avec des agents tel que l'alloxane ou la streptozotocine (Wolfran et al., 2007; Hanhineva et al., 2010).

Dans cette étude, il s'agit d'une induction temporaire par une solution de glucose. Les résultats obtenus (Tableau 3) montrent que les souris qui ont consommé les extraits EED, EAE et EBB indiquent respectivement un pourcentage de réduction de glycémie de $51,1 \% ; 48,5 \%$ et $7,5 \%$ par rapport au contrôle négatif deux heures après prise des extraits. L'extrait aqueux des fruits $R$. gentiliana montrent la valeur la plus élevée de réduction de la valeur de glycémie par rapport au contrôle négatif soit de 52,6\%. Une valeur comparable à celle du contrôle positif (glibenclamide) soit 52,5\%. En effet, les composés hydrosolubles autres que les tannins (flavonoïdes, saponines, alcaloïdes...) contenus dans l'extrait EAQ apporteraient un supplément d'activité antihyperglycémique. Ces résultats confortent ceux obtenus dans une étude antérieure par notre équipe de recherche indiquant les valeurs de l'indice et charge glycémiques du fruit entier de $R$. gentiliana particulièrement basses, justifiant ainsi l'utilisation de ce fruit comme hypoglycémiant par la population congolaise (Mpiana et al., 2013).

En ce qui concerne les mécanismes d'action des polyphénols en général et des tanins en particulier sur le glucose, les études in vitro effectuées sur les vésicules membranaires de la bordure en brosse des intestins des petits lapins (Shimizu et al., 
2000 ; Ovaskainen et al., 2008) ont montré que le gallate d'épicatéchine extrait de thé vert avait une activité hypoglycémiante par un mécanisme d'empêchement de l'absorption du glucose. D'autres études ont rapporté que les tanins en général peuvent agir sur le glucose par divers mécanismes notamment une meilleure absorption du glucose dans le tissus périphérique, la stimulation de la sécrétion de l'insuline des cellules $\beta$ du pancréas, la diminution de la glycation des protéines circulantes, notamment l'hémoglobine glyquée marqueur de l'état glycémique sur le long terme du diabète de type 2 (De boeck et al., 2012).

\section{Activité antihyperglycémique et activité antiradicalaire}

Il est connu que lorsqu'il s'installe au sein de l'organisme un stress oxydatif suite à une hyperglycémie, un antioxydant est susceptible de détruire les radicaux libres et d'améliorer la sécrétion de l'insuline (Rosen et al., 2001).

L'étude de l'activité antihyperglycémique et de l'activité antiradicalaire des extraits de $R$. gentiliana a montré qu'il y avait une meilleure activité antihyperglycémique pour les extraits qui ont les valeurs d'indices de l'efficacité antiradicalaire (EAR) classés comme intermédiaires.

Nous avons alors réalisé une étude de corrélation de Pearson entre l'activité antihyperglycémique et l'activité antiradicalaire à travers les paramètres de réduction de la glycémie exprimés en pourcentage et les valeurs des mesures de l'indice de l'efficacité antiradicalaire (EAR).

Il ressort de cette étude qu'il y a plutôt une corrélation négative significative $(-0,998)$ au niveau 0,01(bilatérale) entre le pourcentage de réduction de la glycémie et les valeurs de l'indice de l'efficacité antiradicalaire. L'extrait qui a une activité antiradicalaire élevée (EBB) n'est pas nécessairement celle qui a une activité antihyperglycemainte élevée.

Il est néanmoins important de remarquer que tous les extraits qui ont une activité antihyperglycémiante élevée ont également une activité antiradicalaire notable (au moins de niveau intermediaire). Ce qui indique qu'ils peuvent agir comme hypoglycémiant et lutter en même temps contre les complications liées à l'hyperglycémie. Ces propriétés ont été observées aussi pour un antidiabétique oral, le glibenclamide qui, en plus de son action insulino sécretagogue possède un effet antiradicalaire (Neri et al., 2005).

\section{Conclusion}

Le présent travail est élaboré dans le cadre d'un programme de recherche sur les plantes médicinales congolaises ayant des propriétés hypoglycémiantes dont le genre Raphia.

Il ressort des résultats obtenus que l'extrait butanol/butanone des tanins possède une forte activité antiradicalaire avec une cinétique rapide. Les extraits acétate d'éthyle, éther diéthylique et aqueux possèdent des activités antiradicalaires intermédiaires avec une cinétique relativement moins rapide. Leurs activités antiradicalaires sont comparables à celle de gliben clamid, un hypoglycémiant que nous avons utilisé comme contrôle positif.

L'activité antihyperglycémique des extraits réalisé in vivo sur un model animale (souris NMRI) a montré que l'extrait aqueux est plus efficace que les autres extraits. Les résultats obtenus indiquent qu'il n'existe pas une corrélation positive entre l'activité antiradicalaire et l'activité antihyperglycémique. Néanmoins, les extraits ayant une activité antihyperglycémiante élevée ont également une activité antiradicalaire notable indiquant que les extraits de ce fruit peuvent agir en même temps sur l'hyperglycémie et ses complications.

Des études sont en cours pour isoler les molécules de ces extraits et tester leurs activités. 


\section{REFERENCES}

Akhtar MS, Iqbal J. 1991. Evaluation de l'effet hypoglycémiant de l'Achyranthes aspera chez des lapins normaux et alloxane-diabétique. J. Ethnopharmacol., 31(1): 49- 57.

Bruneton J.2009. Pharmacognosie, Phytochimie de Plantes Médicinales; (4ème edn, revue et augmenté). Tec \& Doc - Éditions Médicales Internationales : Paris.

De boeck M, Jose G, Derrai K, Wayne S. 2012. Polyphenol and glucose homeostasis in humans. J. Academy Nutrition Dietetics, 112(6): 808-815.

Hanhineva K, Tunoner R, Bon Pon I, Pekkinen J, Kokhannina H, Poutanen K. 2010. Impact of dietary polyphenol on carbohydrate metabolism. J. Mol. Sci., 11: $1365-1402$.

Katemo M, Mpiana PT, Mbala BL, Mihigo So, Ngbolua KN, Tshibangu DST, Koyange PR. 2012. Ethnopharmacological survey of plants used against diabetes in Kisangani city (DR Congo). $J$. Ethnopharmacol., 144: 39-43.

Lacopini P, Baldi M, Storchi P, Sebastiani L.2008. Catechin, epicatechin, quercetin, rutin and resveratrol in red grape: content, in vitro antioxidant activity and interactions. Journal of Food Composition and Analysis, 21: 589-598.

Lathan P, Konda KM. 2006. Quelques Plantes Utiles du Bas-Congo province. Revue des Plantes Médicinales Editions : Kinshasa.

Macheix J-J, Fleuriet A, Jay-Allemand C. 2005. Les Composés Phénoliques des Végétaux: un Exemple de Métabolites Secondaires d'Importance Economique. Presses Polytechniques et Universitaires Romandes : Lausanne, Suisse.

Mpiana PT, Masunda A, Longoma F, Tshibangu DST, Ngbolua KN. 2013. Antihyperglycemic activity of Raphia gentiliana De Wild. European Journal of Medicinal Plants, 3(2): 233-240.

Muanda NF. 2010. Identification de polyphenols, évaluation de leurs activités antioxydante et étude de leurs propriétés biologiques, thèse de doctorat, Université Paul verlaine-Metz, p.104.

Neri S, Signorelli SS, Torrisi B, Pulvirenti D, Mauceri B, Abate G, Ignaccolo L, Bordonaro F, Cilio D, Calvagno S, Leotta C. 2005. Effects of antioxidant supplementation on postprandial oxidative stress and endothelial dysfunction: a single-blind, 15-day clinical trial in patients with untreated type 2 diabetes, subjects with impaired glucose tolerance, and healthy controls. Clin. Ther ., 27: 1764-1773.

Ngbolua KN, Bishola TT, Mpiana PT, Mudogo V, Sha-Tshibey D. Tshibangu DST, Ngombe KN, Ekutsu EG, Tshilanda DD, Gbolo BZ, Mwanangombo DT, Fatiany PR, Baholy R. 2014. Ethno-botanical survey, in vitro antisickling and free radical scavenging activities of Garcinia punctata Oliv. (Clusiaceae). Journal of Advanced Botany and Zoology, 1(2): 1-7.

Ovaskainen MM, Tunoner L, Koponen JH, Sisko H, Hellstra J, Reinivuo H, Mattila P. 2008. Dietary intake and major food sources of polyphenols in finish adults. $J$. Mol Sci, 138: 562-580.

Pitocco D, Martini F, Scavone G, Zaccardi F, Ghirlanda G. 2014. Oxidative Stress and Diabetes in Systems Biology of Free Radicals and Antioxidants, Laher I (ed). Springer: Berlin; 3283-331.

Prakash D, Upadhymy G, Brahma N, Singk HB. 2007. Antioxidant and free radical scaveging activity of seeds and of games varieties of say sean. Food Chemistry, 184: 783-790.

Rani M, Yadav S, Choudhary S, Sharma S, Mani Pandey SM.2014. Effect of glimepiride compared with glibenclamide on post prandial blood sugar in type II diabetes mellitus patients. Int. J. Basic Clin. Pharmacol., 3(1): 96-99.

Ripsin CM, Kang H, Urban RJ. 2009. Management of blood glucose in type 2 diabetes mellitus. Am. Phamily Physician, 79(1): 29-36. 
Riserus U, Willet W, Hu FB. 2009. Dietary fats and prevention of type 2 diabetes, Prog Lipid Res., 48(1): 44 - 49.

Rosen P, Nawroth P, King G, Moller G, Packer L. 2001. The role of oxidative stress in the on set and progressive of diabetes and its complications. Diabetes Metab., 17: 189-212.

Sanchez-Moreno C, Larrauri Jose A, SauraCalixto FA. 1998. Procedure to Measure the Antiradical Efficiency of Polyphenols. Journal of the Science of Food and Agriculture, 76(2): 270-276.

Scherer R, Godoy HT. 2009. Human Antioxidant activity index (AAI) by the 2,2-diphenyl-1-picrylhydrazyl method. Food Chemistry, 112: 654-658.
Sharma Om P, Bhat TK. 2009. DPPH antioxidant assay revisited. Food Chemistry, 113(4): 1202-1205.

Shimizu M, Kobayashi Y, Suzuki M, Satsu H, Miyamoto Y. 2000. Regulation of intestinal glucose transport by tea catechins. Biofactors, 13: 61-65.

Wolfran S. 2007. Effect of green tea and ECG in cardiovascular and metabolc health. J. Am. Coll. Med., 26: 379-388.

Zhang XF, Tan BK. 2000. Propriété Antidiabétique de l'extrait éthanolique de Paniculata chez les rats rendus diabétiques à la streptozotocine. Andrographis, Acta Sin. Pharmacology, 21: 157-164. 